\title{
Time-Dependent Nonplanar Dust-Ion-Acoustic Gardner Double Layers
}

\author{
Farah Deeba*, A. A. Mamun \\ Department of Physics, Jahangirnagar University, Dhaka, Bangladesh \\ E-mail: 'deeba.ju35@yahoo.com \\ Received September 24, 2011; revised October 30, 2011; accepted November 10, 2011
}

\begin{abstract}
A theoretical investigation has been made on the nonplanar (cylindrical and spherical) dust-ion-acoustic (DIA) double layers (DLs) in a dusty plasma system, containing inertial ions, Boltzmann electrons, and negatively charged stationary dust. In this investigation, in order to analyze the time dependent nonplanar DIA DLs, we have used the modified Gardner equation, which has been obtained by employing the reductive perturbation method. It has been found that the behaviors of DIA DLs have been significantly modified by the time period and the nonplanar geometry. The nonplanar DIA DLs has been found to be similar with planar DIA DLs only at large time scale and the cylindrical DIA DLs have been found to be smaller than the spherical DLs, but larger than the planar DLs.
\end{abstract}

Keywords: Dust-Ion-Acoustic, Double Layers, Nonplanar

\section{Introduction}

The existence of novel dust-ion-acoustic (DIA) waves was first predicted by Shukla and Silin [1] about twenty years ago. Nearly four years later, the prediction of Shukla and Silin [1] was conclusively verified by a laboratory experiment of Barkan et al. [2]. Then the linear features of the DIA waves have been rigorously investigated by a number of authors $[1,3,4]$. The linear properties of the DIA waves in dusty plasmas are now well understood from both theoretical and experimental points of view [1-5].

The nonlinear structures (viz., solitary waves, shock structure, and double layers) associated with the DIA waves have also received a great deal of interest because they have a great impact in understanding the basic properties of the localized electrostatic perturbations not only in space [6-9], but also in laboratory dusty plasmas [10-14]. A number of investigations have been made on these nonlinear structures [6-12], particularly DIA solitary waves (SWs) [11,15-19], shock waves [12,13,20-24], and double layers (DLs) [25-28]. All of these work [11, 12,15-19] are confined in planar geometry. Since the waves observed in laboratory devices are certainly not bounded in one-dimension, the investigations made on 1D (planar) nonlinear DIA waves, may not be appropriate for realistic space or laboratory dusty plasma situa- tions. Recently, a few investigations have been made on finite amplitude nonplanar DIA solitary and shock structures [29-31]. But in all of these investigations [29-31], authors have used the $\mathrm{K}-\mathrm{dV}$ or Burgers equations, which are not valid for a parametric regime corresponding to $A$ $=0$ or $A \sim 0$ (where $A$ is the coefficient of the nonlinear term of the $\mathrm{K}-\mathrm{dV}$ or Burger equation [29-31], and $A \sim 0$ means here that $A$ is not equal 0 , but $A$ is around 0 ). This is because, the latter gives rise to infinitely large amplitude structures which break down the validity of the reductive perturbation method [32].

On the other hand, to the best of our knowledge, no attempt has been made on nonplanar DIA DLs. Therefore, in our present work we are going to analyze the nonplanar DIA DLs in dusty plasma system by deriving the modified Gardner $(\mathrm{mG})$ equation.

\section{Basic Equations}

We consider the nonlinear propagation of the DIA waves in an unmagnetized nonplanar (cylindrical and spherical) dusty plasma, consisting of inertial ions, Boltzmann electrons, and negatively charged stationary dust. Thus, at equilibrium we have $n_{i 0}=n_{e 0}+Z_{d} n_{d 0}$, where $Z_{d}$ is the number of electrons residing onto the dust grain surface, and $n_{d 0}\left(n_{i 0}\right)$ is the dust (ion) number density at equilibrium. The nonlinear dynamics of the DIA waves, 
whose phase speed is much smaller (larger) than the electron (ion) thermal speed, in a nonplanar geometry is governed by

$$
\begin{gathered}
\frac{\partial n_{i}}{\partial t}+\frac{1}{r^{v}} \frac{\partial}{\partial r}\left(r^{v} n_{i} u_{i}\right)=0, \\
\frac{\partial u_{i}}{\partial t}+u_{i} \frac{\partial u_{i}}{\partial r}=-\frac{\partial \phi}{\partial r}, \\
\frac{1}{r^{v}} \frac{\partial}{\partial r}\left(r^{v} \frac{\partial \phi}{\partial r}\right)=-\rho, \\
\rho=n_{i}-(1-\mu) \exp (\phi)-\mu,
\end{gathered}
$$

where $v=0$ for $1 D$ planar geometry, and $v=1(2)$ for a nonplanar cylindrical (spherical) geometry; $n_{i}$ is the ion number density normalized by its equilibrium value $n_{i 0} ; u_{i}$ is the ion fluid speed normalized by

$C_{i}=k_{\mathrm{B}} T_{e} / m_{i} ; \phi$ is the electrostatic wave potential normalized by $k_{B} T_{e} / e, \rho$ is the surface charge density normalized by $k_{B} T_{e} / e ; \mu=Z_{d} n_{d 0} / n_{i 0}$, is the ratio of dust number density to the ion number density (the quantity $\mu$ is often used to measure the quantity of dust particles present in the plasma system); $m_{i}\left(m_{e}\right)$ is the mass of the ion (electron); $T_{i}\left(T_{e}\right)$ is the ion (electron) temperature, $Z_{d}$ is the number of electrons residing on a dust grain surface; $k_{B}$ is the Boltzmann constant, and $e$ is the magnitude of the electron charge. The time and space variables are in units of the ion plasma period $\omega_{p i}^{-1}=\left(m_{i} / 4 \pi n_{i 0} \mathrm{e}^{2}\right)^{1 / 2}$ and the Debye radius $\lambda_{D m}=\left(k_{B} T_{e} / 4 \pi n_{i 0} \mathrm{e}^{2}\right)^{1 / 2}$, respectively.

\section{Derivation of $\mathrm{mG}$ Equation}

To study finite amplitude DIA DLs by employing the reductive perturbation method $[27,33,34]$, we first introduce the stretched coordinates:

$$
\begin{gathered}
\zeta=\varepsilon\left(r-V_{p} t\right), \\
\tau=\varepsilon^{3} t,
\end{gathered}
$$

where $\varepsilon$ is a smallness parameter $(0<\varepsilon<1)$ measuring the weakness of the dispersion, and $V_{p}$ (normalized by $C_{i}$ ) is the phase speed of the perturbation mode. We then expand all the dependent variables (viz. $n_{i}, u_{i}, \phi$, and $\rho$ ) in power series of $\varepsilon$ :

$$
\begin{aligned}
& n_{i}=1+\varepsilon n_{i}^{(1)}+\varepsilon^{2} n_{i}^{(2)}+\varepsilon^{3} n_{i}^{(3)}+\cdots \\
& u_{i}=0+\varepsilon u_{i}^{(1)}+\varepsilon^{2} u_{i}^{(2)}+\varepsilon^{3} u_{i}^{(3)}+\cdots \\
& \phi=0+\varepsilon \phi^{(1)}+\varepsilon^{2} \phi^{(2)}+\varepsilon^{3} \phi^{(3)}+\cdots \\
& \rho=0+\varepsilon \rho^{(1)}+\varepsilon^{2} \rho^{(2)}+\varepsilon^{3} \rho^{(3)}+\cdots
\end{aligned}
$$

Now, Expressings (1)-(4) in terms of $\zeta$ and $\tau$, and substituting (7)-(10) into the resulting [Equations (1)-(4) expressed in terms of $\zeta$ and $\tau$ ], one can easily develop different sets of equations in various powers of $\varepsilon$. To the lowest order in $\varepsilon$, one obtains

$$
\begin{aligned}
& u_{i}^{(1)}=\frac{\psi}{V_{p}}, n_{i}^{(1)}=\frac{\psi}{V_{p}^{2}}, \\
& \rho^{(1)}=0, V_{p}^{2}=\frac{1}{\mu_{e}},
\end{aligned}
$$

where $\psi=\phi^{(1)}$ and $\mu_{e}=1-\mu$. The expression for $V_{p}^{2}$ in (12) represents the linear dispersion relation for the DIA waves propagating in a dusty plasma under consideration. This equation clearly indicates that the DIA wave phase speed $\left(V_{p}\right)$ increases with the increase of the dust charge density $\left(Z_{d} n_{d 0}\right)$. To the next higher order in $\varepsilon$, we obtain a set of equations, which, after using (11) and (12), can be simplified as

$$
\begin{gathered}
u_{i}^{(2)}=\frac{\psi^{2}}{2 V_{p}^{3}}+\frac{\phi^{(2)}}{V_{p}}, n_{i}^{(2)}=\frac{3 \psi^{2}}{2 V_{p}^{4}}+\frac{\phi^{(2)}}{V_{p}^{2}}, \\
\rho^{(2)}=\frac{1}{2} A \psi^{2}=0, A=(1-\mu)(2-3 \mu) .
\end{gathered}
$$

It is obvious from (14) that $A=0$ since $\psi \neq 0$. Now by solving $A=0$ for $\mu$, we found $A$ can be zero for both $\mu=1$ and $\mu=3 / 2$. But when $\mu=1, Z_{d} n_{d 0}$ (dust number density at equilibrium) will be equal to $n_{i 0}$ (ion number density at equilibrium), which means that there is no electron present in the system, i.e., all electrons will be captured by dust particles. As our model contains all of electron, ion, and dust, this is an invalid condition for our present model. For our system $\mu<1$. Therefore the only valid solution of $A=0$ for $\mu$ is given by

$$
\mu=\mu_{c}=2 / 3 .
$$

For $\mu$ around its critical value $\left(\mu_{c}\right)$, i.e. for $\left|\mu-\mu_{c}\right|=\varepsilon$ corresponding to $A=A_{0}$, we can express $A_{0}$ as

$$
A_{0} \simeq s\left(\frac{\partial A}{\partial \mu}\right)_{\mu=\mu_{c}}\left|\mu-\mu_{c}\right|=s \varepsilon .
$$

where $\left|\mu-\mu_{c}\right|$ is a small and dimensionless parameter, and can be taken as the expansion parameter $\varepsilon$, i.e. $\left|\mu-\mu_{c}\right| \simeq \varepsilon$, and $s=1$ for $\mu<\mu_{c}$ and $s=-1$ for $\mu>\mu_{c}$. So, $\rho^{(2)}$ can be expressed as

$$
\rho^{(2)} \simeq \frac{1}{2} \varepsilon s \psi^{2},
$$

This means that for $\mu \neq \mu_{c}, \rho^{(2)}$ must be included in the third order Poisson's equation. To the next higher order in $\varepsilon$, we obtain a set of equations: 


$$
\begin{gathered}
\frac{\partial \psi}{\partial \tau}-V_{p}^{3} \frac{\partial n_{i}^{(3)}}{\partial \zeta}-V_{p}^{2} \frac{\partial u_{i}^{(3)}}{\partial \zeta}+\frac{2}{V_{p}^{3}} \frac{\partial \psi^{3}}{\partial \zeta} \\
+\frac{2}{V_{p}} \frac{\partial}{\partial \zeta}\left[\psi \varphi^{(2)}\right]+\frac{\nu \psi}{\tau}=0, \\
\frac{\partial \psi}{\partial \tau}+V_{p}^{2} \frac{\partial u_{i}^{(3)}}{\partial \zeta}+\frac{1}{2 V_{p}^{3}} \frac{\partial \psi^{3}}{\partial \zeta}+\frac{1}{V_{p}} \frac{\partial}{\partial \zeta}\left[\psi \varphi^{(2)}\right] \\
+V_{p} \frac{\partial \varphi^{(3)}}{\partial \zeta}=0, \\
\frac{\partial^{2} \psi}{\partial \zeta^{2}}+n_{i}^{(3)}-\mu_{e} \phi^{(3)}-\mu_{e} \psi \phi^{(2)}-\frac{1}{6} \mu_{e} \psi^{3} \\
+\frac{s}{2} \psi^{2}=0 .
\end{gathered}
$$

Now, using (11)-(14) and (18)-(20), we finally obtain a nonlinear dynamical equation of the form:

$$
\frac{\partial \psi}{\partial \tau}+\frac{v}{2 \tau} \psi+s \beta \psi \frac{\partial \psi}{\partial \zeta}+\alpha \beta \psi^{2} \frac{\partial \psi}{\partial \zeta}+\beta \frac{\partial^{3} \psi}{\partial \zeta^{3}}=0,
$$

where

$$
\begin{gathered}
\alpha=\frac{15}{2}(1-\mu)\left\{(1-\mu)^{2}-\frac{1}{15}\right\}, \\
\beta=\frac{1}{2}(1-\mu)^{-\frac{3}{2}} .
\end{gathered}
$$

Equation (21) is known as modified Gardner (mG) equation. The modification is due to the extra term, $\frac{v}{2 \tau} \psi$, which arises due to the effects of the nonplanar geometry. Because of the existence of both $\psi^{2}$ and $\psi^{3}$ term, this equation supports both Sws and DLs solutions. We have already mentioned that $v=0$ corresponds to a $1 D$ planar geometry which reduces (21) to a standard Gardner (sG) equation. We are now going to numerically analyze the $\mathrm{mG}$ equation. However, for a better understanding, before going to numerical solutions of $\mathrm{mG}$ equation, we first briefly discuss the stationary double layer (DL) solution of this standard Gardner equation [i.e. (21) with $v=0$ ]. The stationary DL solution of the sG equation [i.e. (21) with $v=0$ ] is obtained by considering a moving frame (moving with speed $U_{0}$ ) $\xi=\zeta-U_{0} \tau$, and imposing all the appropriate boundary conditions for DL solution, including $\psi \rightarrow 0$,

$\mathrm{d} \psi / \mathrm{d} \xi \rightarrow 0, \mathrm{~d}^{2} \psi / \mathrm{d} \xi^{2} \rightarrow 0$ at $\xi \rightarrow-\infty$. These boundary conditions for the stationary DL solution allow us to express the $\mathrm{sG}$ equation as

$$
\frac{1}{2}\left(\frac{\mathrm{d} \psi}{\mathrm{d} \xi}\right)^{2}+V(\psi)=0
$$

where the pseudo-potential $V(\psi)$ is

$$
V(\psi)=-\frac{U_{0}}{2 \beta} \psi^{2}+\frac{s}{6} \psi^{3}+\frac{\alpha}{12} \psi^{4} .
$$

We note here that $U_{0}$ and $\beta$ are always positive since $\mu \leq 1$ is always valid. It is obvious from (25) that

$$
\begin{gathered}
\left.V(\psi)\right|_{\psi=0}=\left.\frac{\mathrm{d} V(\psi)}{\mathrm{d} \psi}\right|_{\psi=0}=0, \\
\left.\frac{\mathrm{d}^{2} V(\psi)}{\mathrm{d} \psi^{2}}\right|_{\psi=0}<0 .
\end{gathered}
$$

The conditions (26) and (27) imply that DL solution of (24) exist if and only if

$$
\left.V(\psi)\right|_{\psi=\psi_{m}}=\left.\frac{\mathrm{d} V(\psi)}{\mathrm{d} \psi}\right|_{\psi=\psi_{m}}=0 .
$$

where $\psi_{m}$ is the amplitude of the DLs. The latter can be expressed as

$$
\begin{aligned}
& U_{0}=-\frac{s^{2} \beta}{6 \alpha}, \\
& \psi_{m}=\frac{6 U_{0}}{s \beta} .
\end{aligned}
$$

Now, using (25) and (30) in (24) we have

$$
\left(\frac{\mathrm{d} \psi}{\mathrm{d} \xi}\right)^{2}+\gamma \psi^{2}\left(\psi-\psi_{m}\right)^{2}=0,
$$

where $\gamma=\alpha / 6$. Now, integrating (31) the stationary DL solution of sG equation [i.e. (21) with $v=0$ ] can be directly given by

$$
\psi=\frac{\psi_{m}}{2}\left[1+\tanh \left(\frac{\xi}{\Delta}\right)\right],
$$

where $\Delta$ is the DL width of the DLs, and is given

$$
\Delta=\frac{2}{\psi_{m} \sqrt{-\gamma}}
$$

From (32) and (33), it is clear that DLs can be formed in the dusty plasma system if and only if $\alpha<0$, i.e. $\mu_{L}<\mu<\mu_{U}$, where $\mu_{L}=0.74 \quad\left(\mu_{U}=1\right)$, obtained from $\alpha=0$, is the lower (upper) limit of $\mu$ above (below) which DLs exist.

In Figures 1 and 2, the variations of $\psi_{m}$ with $U_{0}$ (Figure 1) and $\mu_{e}$ (Figure 2) have been graphically shown. On the other hand, since $\beta>0$ and $\gamma>0$, (32) and (33) indicate that the DLs are associated with positive potential if $s=1$, i.e. $\mu<\mu_{c}$, and associated with negative potential if $s=-1$, i.e. $\mu>\mu_{c}$. It is obvious from Figures 1-3 that $\mu_{L}>\mu_{c}$ which indicates that our DLs are associated only with negative potential. The parametric regimes for the existence of negative 


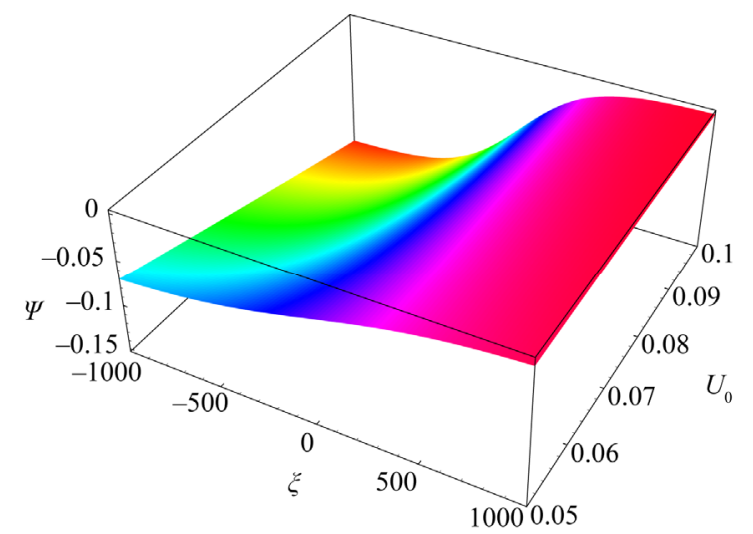

Figure 1. Showing the variation of the amplitude of DIA DLs with $U_{0}$ at $\mu=0.75$.

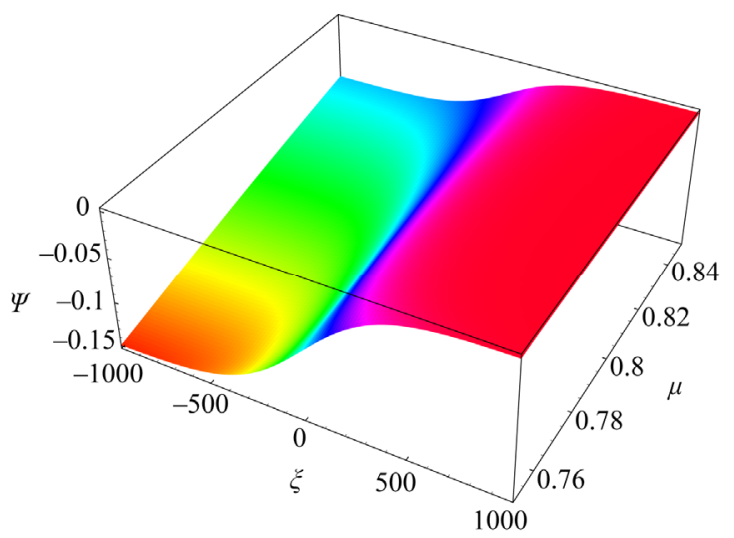

Figure 2. Showing the variation of the amplitude of DIA DLs with $\mu$ at $U_{0}=0.1$.

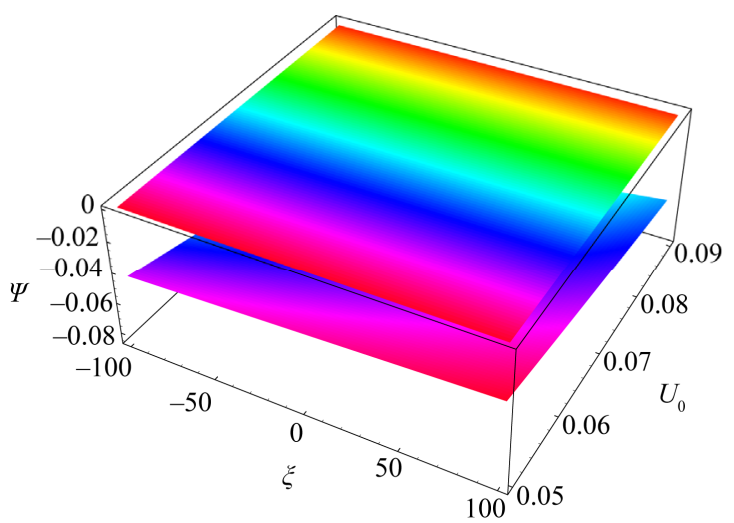

Figure 3. Showing the parametric regime for the existence of DLs. The upper (lower) surface plot represents the lower (upper) limit of $\mu$ below (above) which DLs exist.

DLs are bounded by the lower and upper surface plot of Figure 3, and DLs exist for parameters corresponding to any point in between two $(\alpha=0)$ surface plots.

The point to be noted here that if we would keep only the lower order nonlinear term of (21) (viz. the third term of (21) or the term containing $\psi^{2}$ ) instead of the higher order nonlinear term (viz. the fourth term of (21) or the term containing $\psi^{3}$ ), we would obtain the solitary structures. On the other hand, in our present work, we have kept both the terms containing $\psi^{2}$ and $\psi^{3}$, and have obtained the DL structures (associated with $\psi^{2}$ and $\left.\psi^{3}\right)$.

\section{Numerical Analysis}

Now in order to analyze the nonplanar DIA DLs, we turn to $(21)$ with the term $(v / 2 \tau) \psi$, which is due to the effects of the nonplanar (cylindrical or spherical) geometry. An exact analytic solution of (21) is not possible. Therefore, we have numerically solved (21), and have studied the effects of cylindrical and spherical geometries on time-dependent DIA DLs. The results are depicted in Figures 4 and 5. The initial condition, which we have used in numerical analysis, is in the form of the stationary solution of $(21)$ without the term $(v / 2 \tau) \psi$. Figure 4 shows how the effects of a cylindrical geometry modify the DIA DLs, and Figure 5 shows how the effects of a spherical geometry modify the DIA DLs.

From the numerical solutions of (21) (displayed in Figures 4 and 5) we may conclude that for a large value of $\tau$ (e.g. $\tau=-40)$, the cylindrical $(v=1)$ and spherical $(v=2)$ DLs are almost similar to $1 D$ planar $(v=0)$ structures. This is because when the value of $\tau$ is large, the term $(v / 2 \tau) \psi$, which is due to the effects of the cylindrical or spherical geometry, is no longer dominant. However, as the value of $\tau$ decreases, the term $(v / 2 \tau) \psi$ becomes dominant, and spherical and cylindrical DL structures differ from $1 D$ (planar) ones. It has been observed that as the value of $\tau$ decreases, the amplitude of these localized pulses increases. It is also found that the amplitude of cylindrical DIA DL structures is larger than those of $1 D$ planar ones, but smaller than that of the spherical ones.

\section{Discussion and Conclusions}

In this paper we have investigated time-dependent nonplanar dust-ion-acoustic Gardner double layers in a dusty plasma system (composed of inertial ions, Boltzmann electrons, and negatively charged stationary dust), by deriving modified Gardner ( $\mathrm{sG}$ ) equation. The outcomes, which have been obtained from this investigation can be pinpointed as follows:

1) The dusty plasma system under consideration supports both finite amplitude planar and nonplanar DLs, whose basic features (polarity, amplitude, width, etc.) depend on the ion and dust number densities.

2) The DLs having large width exist for $0.74<\mu<1$ and only have negative potential, i.e., no positive DLs 


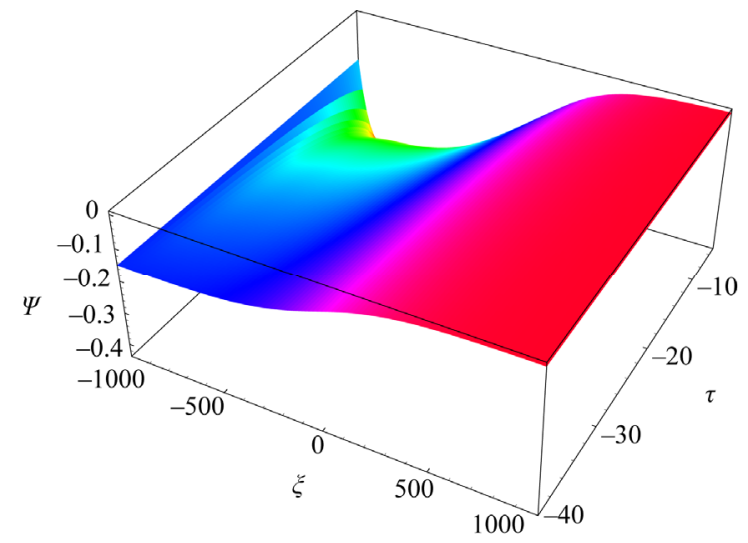

Figure 4. Showing the effects of cylindrical geometry on DIA DLs at $\boldsymbol{\mu}=\mathbf{0 . 7 5}$.

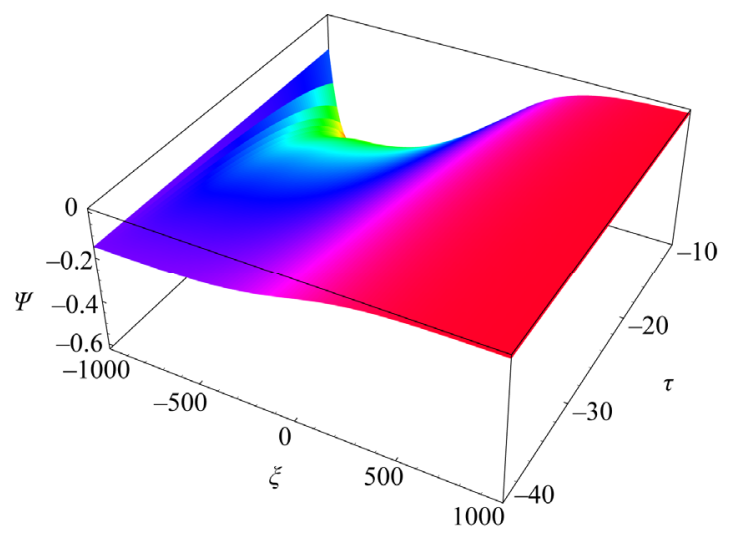

Figure 5. Showing the effect of spherical geometry on DIA DLs at $\mu=0.75$.

have been formed.

3) The magnitude of the amplitude of the DLs increases with the increase of $U_{0}$, but decreases with the increase of $\mu$.

4) The magnitude of the amplitudes of both cylindrical and spherical DLs increase with decrease of $\tau$.

5) The spherical DLs have larger amplitude and potential than the cylindrical and planar DLs.

We, finally hope that our results may be useful in understanding the localized electrostatic disturbances in both space environments [6-9], and laboratory devices [11-14].

\section{Acknowledgements}

The Third World Academy of Science (TWAS) Research Grant for research equipment is gratefully acknowledged.

\section{References}

[1] P. K. Shukla and V. P. Silin, "Dust Ion-Acoustic Wave,"
Physica Scripta, Vol. 45, No. 5, 1992, p. 508. doi:10.1088/0031-8949/45/5/015

[2] A. Barkan, N. D'Angelo and R. L. Merlino, "Experiments on Ion-Acustic Waves in Dusty Plasmas," Planetary and Space Science, Vol. 44, No. 1, 1996, pp. 239-242. doi:10.1016/0032-0633(95)00109-3

[3] P. K. Shukla and M. Rosenberg, "Boundary Effects on Dust-Ion-Acoustic and Dust-Acoustic Waves in Collisional Dusty Plasmas," Physics of Plasmas, Vol. 6, No. 3, 1999, pp. 1038-1040. doi:10.1063/1.873345

[4] P. K. Shukla and A. A. Mamun, "Introduction to Dusty Plasma Physics," Institute of Physics Publishing Ltd., Bristol, 2002. doi:10.1887/075030653X

[5] R. L. Merlino and J. Goree, "Dusty plasmas in the laboratory, Industry, and Space," Physics Today, Vol. 57, No. 7, 2004, pp. 32-39. doi:10.1063/1.1784300

[6] V. E. Fortov, et al., "Complex (Dusty) Plasmas: Current Status, Open Issues, Perspectives," Physics Report, Vol. 421, No. 1-2, 2005, pp. 1-103. doi:10.1016/j.physrep.2005.08.007

[7] O. Ishihara, "Complex Plasma: Dusts in plasma," Journal of Physics D, Vol. 40, No. 8, 2007, p. R121. doi:10.1088/0022-3727/40/8/R01

[8] P. K. Shukla and B. Eliasson, "Colloquium: Fundamentals of Dust-Plasma Interactions," Reviews of Modern Physics, Vol. 81, No. 1, 2009, pp. 25-44. doi:10.1103/RevModPhys.81.25

[9] G. E. Morfill and A. V. Ivlev, "Complex plasmas: An Interdisciplinary Research Field," Reviews of Modern Physics, Vol. 81, No. 4, 2009, pp. 1353-1404. doi:10.1103/RevModPhys.81.1353

[10] K. E. Lonngren, "Soliton Experiments in Plasmas," Plasma Physics, Vol. 25, No. 9, 1983, p. 943. doi:10.1088/0032-1028/25/9/001

[11] Y. Nakamura and A. Sharma, "Observation of Ion-Acoustic Solitary Waves in a Dusty Plasma," Physics of Plasmas, Vol. 8, No. 9, 2001, pp. 3921-3926. doi:10.1063/1.1387472

[12] Y. Nakamura, H. Bailung and P. K. Shukla, "Observation of Ion-Acoustic Shocks in a Dusty Plasma," Physical Reviews Letters, Vol. 83, No. 8, 1999, pp. 1602-1605. doi:10.1103/PhysRevLett.83.1602

[13] Q. Z. Luo, N. D'Angelo and R. L. Merlino, "Experimental Study of Shock Formation in a Dusty Plasma," Physics of Plasmas, Vol. 6, No. 9, 1999, pp. 3455-3458. doi:10.1063/1.873605

[14] C. Charles, "A Review of Recent Laboratory Double Layer Experiments," Plasma Sources Science and Technology, Vol. 16, No. 4, 2007, pp. R1-R25. doi:10.1088/0963-0252/16/4/R01

[15] R. Bharuthram and P. K. Shukla, "Large Amplitude IonAcoustic Solitons in a Dusty Plasma," Planetary and Space Science, Vol. 40, No. 7, 1992, pp. 973-977. doi:10.1016/0032-0633(92)90137-D

[16] S. I. Popel and M. Y. Yu, "Ion Acoustic Solitons in Impurity-Containing Plasmas," Contributions to Plasma Physics, Vol. 35, No. 2, 1995, pp. 103-108. 
doi:10.1002/ctpp. 2150350202

[17] A. A. Mamun and P. K. Shukla, "Cylindrical and Spherical Dust Ionacoustic Solitary Waves," Physics of Plasmas, Vol. 9, No. 4, 1997, pp. 1468-1470.

[18] P. K. Shukla and A. A. Mamun, "Solitons, Shocks and Vortices in Dusty Plasmas," New Journal of Physics, Vol. 5, 2003, pp. 17.1-17.37.

[19] S. I. Popel, et al., "Weakly Dissipative Dust-Ion-Acoustic Solitons," Physical Review E, Vol. 67, No. 5, 2003, p. 056402(5). doi:10.1103/PhysRevE.67.056402

[20] P. K. Shukla, "Dust Ion-Acoustic Shocks and Holes," Physics of Plasmas, Vol. 7, No. 3, 2000, pp. 1044-1046. doi:10.1063/1.873905

[21] S. I. Popel, A. P. Golub and T. V. Losseva, "Dust IonAcoustic Shock-Wave Structures: Theory and Laboratory Experiments," JETP Lettrers, Vol. 74, No. 7, 2001, pp. 362-366. doi:10.1134/1.1427122

[22] A. A. Mamun and P. K. Shukla, "The Role of dust Charge Fluctuations on Nonlinear Dust Ion-Acoustic Waves," IEEE Transactions on Plasma Science, Vol. 30, No. 2, 2002, pp. 720-724. doi:10.1109/TPS.2002.1024274

[23] A. A. Mamun, P. K. Shukla and B. Eliasson, "Arbitrary Amplitude Dust Ion-Acoustic Shock Waves in a Dusty Plasma with Positive and Negative Ions," Physics of Plasmas, Vol. 16, No. 11, 2009, pp. 114503-114506. doi:10.1063/1.3261840

[24] S. S. Duha and A. A. Mamun, "Dust-Ion-Acoustic Shock Waves Due to Dust Charge Fluctuation," Physical Letters A, Vol. 373, No. 14, 2009, pp. 1287-1289. doi:10.1016/j.physleta.2009.01.059

[25] A. A. Mamun, P. K. Shukla and B. Eliasson, "Solitary Waves and Double Layers in a Dusty Electronegative Plasma," Physical Reviews E, Vol. 80, No. 4, 2009, p. 046406(6). doi:10.1103/PhysRevE.80.046406

[26] H. Alinejad and A. A. Mamun, "Small Amplitude Dust Ion-
Acoustic Solitary Waves and Double Layers in a Dusty Plasma with Flat-Topped Electron Distribution," Physics of Plasmas, Vol. 17, No. 12, 2010, p. 123704(5). doi:10.1063/1.3526649

[27] S. Ghosh and R. Bharuthram, "Ion Acoustic Solitons and Double Layers in Electronpositronion Plasmas with Dust Particulates," Astrophysics and Space Science, Vol. 314, No. 1-3, 2008, pp. 121-127. doi:10.1007/s10509-008-9748-0

[28] R. Roychoudhury and P. Chatterjee, "Arbitrary Amplitude Double Layers in Dusty Plasma," Physics of Plasmas, Vol. 6, No. 1, 1999, pp. 406-408. doi:10.1063/1.873294

[29] A. A. Mamun and P. K. Shukla, "Cylindrical and Spherical Dust Ionacoustic Solitary Waves," Physics of Plasmas, Vol. 9, No. 4, 2002, pp. 1468-1470. doi:10.1063/1.1458030

[30] A. A. Mamun and P. K. Shukla, "Effects of Nonthermal Distribution of Electrons and Polarity of Net Dust-Charge Number Density on Nonplanar Dust-Ion-Acoustic Solitary Waves," Physical Reviews E, Vol. 80, No. 3, 2009, pp. 037401(4). doi:10.1103/PhysRevE.80.037401

[31] A. A. Mamun and P. K. Shukla, "Cylindrical and Spherical Dust Ion-Acoustic Shock Waves in Dusty Plasmas," Europhysics Letters, Vol. 87, No. 2, 2009, p. 25001. doi:10.1209/0295-5075/87/25001

[32] H. Washimi and T. Taniuti, "Propagation of Ion-Acoustic Solitary Waves of Small Amplitude," Physical Reviews Letters, Vol. 17, No. 19, 1966, pp. 996-998. doi:10.1103/PhysRevLett.17.996

[33] S. Maxon and J. Viecelli, "Spherical Solitons," Physical Reviews Letters, Vol. 32, No. 1, 1974, pp. 4-6. doi:10.1103/PhysRevLett.32.4

[34] N. C. Lee, "Small Amplitude Electron-Acoustic Double Layers and Solitons in Fully Relativistic Plasmas of TwoTemperature Electrons," Physics of Plasmas, Vol. 16, No. 4, 2009, p. 042316(10). doi:10.1063/1.3121242 